\section{EFEITOS DO TREINAMENTO DE FORÇA A LONGO PRAZO SOBRE PARÂMETROS HEMODINÂMICOS E CONCENTRAÇÃO DE RESISTINA EM MULHERES NA PÓS-MENOPAUSA}

\section{The effects of long term strength training on hemodynamic parameters and resistin level in postmenopausal women}

\author{
Efectos del entrenamiento de fuerza a largo plazo en los \\ parámetros hemodinámicos y concentración de resistina en \\ mujeres posmenopausia
}

\begin{abstract}
RESUMO
Objetivo: Investigar a influência do treinamento de força (TF) sobre a concentração sorológica de resistina e pressão arterial de mulheres na pós-menopausa. Métodos: Estudo longitudinal, realizado na Universidade Federal de São Carlos, do qual participaram 23 mulheres sedentárias na pós-menopausa. O TF apresentou duração de 13 meses (dez/2008 a jan/2010), com duas sessões semanais, cada uma consistindo em três séries de 8-12 repetições máximas e um exercício para cada grupo muscular principal. Foi avaliada a força muscular máxima nos seguintes exercícios: supino, leg press $45^{\circ}$ e flexão do cotovelo em pé. A concentração sérica de resistina foi determinada pelo método ELISA. No processamento estatístico, utilizou-se o ANOVA (com medidas repetidas) para comparar os momentos Pré, 6 meses e 13 meses $(p<0,05)$. Para avaliar as correlações resistina $\times$ pressão arterial, resistina $\times$ força muscular e força $\times$ pressão arterial, utilizou-se o teste de correlação de Pearson. Resultados: As mulheres apresentaram o seguinte perfil antropométrico: $61,33 \pm 3,8$ anos; estatura de 148,5 $\pm 32,7 \mathrm{~cm}$; massa corporal de $67,56 \pm 10,85 \mathrm{~kg}$. O TF induziu a redução da concentração de resistina $(30272,4 \pm 8100,1$ versus $16350,6 \pm 2404,6 \mathrm{pg} / \mathrm{mL})$ e pressão arterial sistólica $(120,5 \pm 11,8$ versus $115,8 \pm 1,6 \mathrm{mmHg})$, e o aumento da força muscular no leg press $45^{\circ}(172,3 \pm 27,3$ versus $348,6 \pm 40,8 \mathrm{~kg})$, supino $(31,9 \pm 4,1$ versus $41,8 \pm 5,6 \mathrm{~kg})$ e flexão do cotovelo (21,0 2,4 versus $26,5 \pm 2,9 \mathrm{~kg}$ ) após os 13 meses $(\mathrm{p}<0,05)$. Conclusão: Os resultados deste estudo revelaram que o TF a longo prazo aumenta a força muscular máxima, diminui a pressão arterial sistólica e os níveis séricos da resistina - alterações fisiológicas benéficas para a redução do risco de doenças cardiovasculares em mulheres na pós-menopausa.
\end{abstract}

Descritores: Programa de Musculação por Levantamento de Peso; Mulheres; Pósmenopausa; Resistina; Pressão Arterial.

\section{ABSTRACT}

Objective: Investigate the influence of strength training (ST) on serum resistin levels and blood pressure of postmenopausal women. Methods: Longitudinal study conducted at the Federal University of São Carlos with twenty-three sedentary postmenopausal women. The ST lasted 13 months (Dec./2008 to Jan./2010) and consisted of two weekly sessions with three sets of 8-12 maximum repetitions and one exercise for each main muscle group. Maximum muscular strength was tested in the following exercises: bench press, $45^{\circ} \mathrm{leg}$ press, and standing arm curl. Serum resistin level was determined using the ELISA method. ANOVA (with repeated measures) was used for the comparisons between periods Pre-, 6 months and 13 months $(p<0.05)$; Pearson's correlation test was used to evaluate the correlations between resistin $\times$ blood pressure, resistin $\times$ muscle strength and strength $\times$ blood pressure. Results: Women presented the following anthropometric profile: $61.33 \pm 3.8$ years; height $148.5 \pm 32.7 \mathrm{~cm}$; body mass $67.56 \pm 10.85 \mathrm{~kg}$. The $S T$ decreased resistin levels $(30272.4 \pm$ 8100.1 to $16350.6 \pm 2404.6 \mathrm{pg} / \mathrm{mL})$ and systolic blood pressure $(120.5 \pm 11.8$ to $115.8 \pm 1.6$ $\mathrm{mmHg})$, and increased muscular strength in the leg press $45^{\circ}(172.3 \pm 27.3$ to $348.6 \pm 40.8$
Artigo Original
1) Centro Universitário Central Paulista UNICEP - São Carlos (SP) - Brasil

2) Universidade Federal da Paraíba - UFPB - João Pessoa (PB) - Brasil

3) Universidade Federal de São Paulo UNIFESP - Baixada Santista - Santos (SP) - Brasil

4) Universidade Católica de Brasília - UCB - Brasília (DF) - Brasil

5) Universidade Federal de São Carlos UFSCar - São Carlos (SP) - Brasil

Recebido em: 12/12/2012 
$\mathrm{kg})$, bench press $(31.9 \pm 4.1$ to $41.8 \pm 5.6 \mathrm{~kg})$ and arm curl $(21.0 \pm$ 2.4 to $26.5 \pm 2.9 \mathrm{~kg})$ after 13 months $(p<0.05)$. Conclusion: The results of this study revealed that long-term $S T$ increases maximum muscular strength, decreases systolic blood pressure and serum resistin levels, which are beneficial physiological alterations that reduce the risk for cardiovascular diseases in postmenopausal women.

Descriptors: Resistance Training Program for Weightlifting; Post-menopausal; Women; Resistin; Arterial Pressure.

\section{RESUMEN}

Objetivo: Investigar la influencia del entrenamiento de fuerza $(E F)$ sobre la concentración serológica de resistina y presión arterial en mujeres posmenopausia. Métodos: Estudio longitudinal realizado en la Universidad Federal de São Carlos en el cual participaron 23 mujeres sedentarias posmenopausia. El EF tuvo duración de 13 meses (diciembre de 2008 a enero de 2010) con dos sesiones semanales, siendo cada sesión constituida de tres series de 8-12 repeticiones máximas y un ejercicio para cada grupo muscular principal. Fue evaluada la fuerza muscular máxima en los siguientes ejercicios: supino, leg press $45^{\circ}$ y flexión de codo de pie. La concentración sérica de resistina fue determinada por el método ELISA. En el procesamiento estadístico se utilizó ANOVA (con medidas repetidas) para las comparaciones entre los momentos pre, 6 meses y 13 meses ( $p<0,05)$; se utilizó la prueba de correlación de Pearson para evaluar las correlaciones entre resistina $\times$ presión arterial, resistina $\times$ fuerza muscular $y$ fuerza $\times$ presión arterial. Resultados: Las mujeres presentaron el siguiente perfil antropométrico: 61,33 $\pm 3,8$ años; altura $148,5 \pm 32,7 \mathrm{~cm}$; masa corporal 67,56 $\pm 10,85 \mathrm{~kg}$. El EF llevó a la reducción de la concentración de resistina $(30272,4 \pm 8100,1$ versus $16350,6 \pm 2404,6 \mathrm{pg} / \mathrm{mL})$ y presión arterial sistólica $(120,5 \pm 11,8$ versus $115,8 \pm 1,6 \mathrm{mmHg}$ ) y aumento de fuerza muscular en el leg press $45^{\circ}(172,3 \pm 27,3$ versus $348,6 \pm 40,8 \mathrm{~kg})$, supino $(31,9 \pm 4,1$ versus $41,8 \pm 5,6 \mathrm{~kg})$ y flexión de codo $(21,0 \pm 2,4$ versus $26,5 \pm 2,9 \mathrm{~kg})$ después de los 13 meses ( $p<0,05)$. Conclusión: Los resultados del estudio revelaron que el EF a largo plazo aumenta la fuerza muscular máxima, disminuye la presión arterial sistólica y los niveles séricos de la resistina, alteraciones fisiológicas benéficas para la reducción del riesgo de enfermedades cardiovasculares en mujeres posmenopausia.

Descriptores: Entrenamiento de Resistencia; Mujeres; Posmenopausia; Resistina; Presión Arterial

\section{INTRODUÇÃO}

$\mathrm{O}$ constante aumento do número de mulheres em relação ao de homens e, principalmente, a sobrecarga de trabalho assumida por elas na sociedade moderna ${ }^{(1)}$ têm ampliado nelas a prevalência de doenças que antes estavam mais relacionadas aos homens, como as cardiovasculares e cerebrovasculares ${ }^{(2)}$. Adicionalmente, o fator idade induz ao surgimento de doenças e distúrbios relacionados ao envelhecimento (câncer de mama, osteoporose, doenças cardiovasculares etc.), comuns em mulheres pós-menopáusicas, estando associados a altos índices de morbidade e mortalidade nesta população( ${ }^{(3)}$.

As mulheres no período pré-menopausa apresentam, em relação aos homens, menor prevalência de hipertensão arterial e doenças afins. No entanto, após a menopausa, ela é similar à dos homens ${ }^{(4)}$. Deficiência de estrógeno, alterações do perfil lipídico, ganho de peso ponderal e sedentarismo estão entre os principais fatores associados à hipertensão arterial em mulheres na menopausa ${ }^{(4)}$.

Um biomarcador importante relacionado a alterações no perfil lipídico, pressão arterial e doenças cardiovasculares é a resistina, uma adipocina recentemente identificada e pertencente a uma família de proteínas ricas em cisteína. A resistina é expressa especificamente no tecido adiposo branco e sua secreção está fortemente relacionada à inflamação, obesidade, resistência à insulina, diabetes, dislipidemias, hipertensão arterial e ao desenvolvimento de doença arterial coronariana ${ }^{(5-7)}$.

Dentre as modalidades de exercícios disponíveis, o treinamento de força (TF) tem sido destacado como responsável por aspectos emocionais, benefícios na densidade mineral óssea e na qualidade de vida, diminuição da dor e da inflamação em mulheres na pós-menopausa ${ }^{(8,9)}$. É recomendado como importante componente em programas de exercícios físicos para indivíduos idosos, promovendo o aumento da força e da massa muscular ${ }^{(10,11)}$. Somando-se a esses fatores, estudos têm demonstrado uma possível associação da força muscular com a diminuição dos fatores de risco cardiovascular ${ }^{(12)}$, obesidade ${ }^{(13)}$, pressão $\operatorname{arterial}^{(14,15)}$, síndrome metabólica ${ }^{(16,17)}$ e morte precoce ${ }^{(18)}$.

No entanto, os efeitos crônicos do TF sobre os níveis plasmáticos da resistina em idosas ainda requerem melhor compreensão $0^{(19)}$. Há necessidade de estudos confirmatórios sobre a eficiência do TF como intervenção terapêutica para a redução do risco cardiovascular, incluindo o biomarcador resistina em idosas ${ }^{(19)}$.

Sendo assim, o objetivo deste estudo foi verificar as respostas crônicas de 13 meses de TF sobre parâmetros hemodinâmicos e concentração de resistina em mulheres na pós-menopausa. A hipótese inicial do estudo era a de que o TF induziria a redução da pressão arterial sistólica (PAS), diastólica (PAD), média (PAM) e pressão de pulso (PP), bem como a concentração de resistina.

\section{MÉTODOS}

O estudo, de natureza quantitativa e longitudinal, ocorreu no período de outubro de 2008 a janeiro de 2010, sendo realizado nos seguintes locais: clínica de fisioterapia 
(avaliações) e academia da UNICEP (treinamento); e laboratório de fisiologia do exercício da UFSCar (análises bioquímicas).

As participantes foram convidadas por meio de campanha de divulgação em panfletos explicativos e reportagens em jornal local. Vinte e três mulheres $(61,33 \pm 3,8$ anos) foram selecionadas e avaliadas clinicamente por um cardiologista (anamnese, aferição dos sinais vitais, exame físico geral, hemograma completo, eletrocardiograma de repouso e esforço, e exame do sistema musculoesquelético). Elas obedeceram aos seguintes critérios de inclusão: período mínimo de três anos de pós-menopausa, ser sedentária (sem atividade física consistente nos seis meses prévios ao estudo), não estar em tratamento de reposição hormonal, não apresentar doenças metabólicas ou endócrinas que afetassem a massa óssea ou muscular e ser cognitivamente capaz de compreender as instruções do treinamento. Também obedeceram aos seguintes critérios de exclusão: alterações musculoesqueléticas (ortopédicas ou reumáticas) que impossibilitassem a execução do protocolo de força proposto, diagnóstico de insuficiência cardíaca congestiva, arritmia severa e hipertensão não controlada.

Inicialmente, as participantes realizaram quatro semanas de familiarização aos exercícios de força. Em seguida, fizeram os testes de uma repetição máxima (1RM) e determinação das cargas das sessões de treinamento. $\mathrm{O}$ programa de TF teve duração de 56 semanas (13 meses), com duas sessões de treinamento por semana (segunda $\mathrm{e}$ quinta-feira) e uma sessão de alongamento geral por semana (quarta-feira). A aferição de pressão arterial sistólica (PAS), pressão arterial diastólica (PAD), pressão arterial média (PAM), pressão de pulso (PP) e resistina sérica ocorreu em três momentos: antes do TF (Pré), 6 meses ( 25 semanas) e 13 meses após o TF.

Determinaram-se a PAS e a PAD pelo método oscilométrico, adotando-se a metodologia proposta pela $\mathrm{V}$ Diretriz Brasileira de Hipertensão Arterial ${ }^{(20)}$ e um medidor oscilométrico (Microlife 3AC1-1, Widnau, Suíça) validado pela European Society of Hypertension. Com a idosa na posição sentada após 10 minutos de repouso, braço direito apoiado ao nível do coração, colocou-se a braçadeira do aparelho (apropriada ao tamanho do braço) a cerca de 3 $\mathrm{cm}$ acima da fossa antecubital, centralizando a bolsa de borracha sobre a artéria umeral. Os valores das medidas da PAS e da PAD foram utilizados para o cálculo da PAM, através da equação: $\mathrm{PAM}=\mathrm{PAD}+[(\mathrm{PAS}-\mathrm{PAD}) \div 3]^{(21)}$. A PP foi determinada pela equação: $\mathrm{PP}=\mathrm{PAS}-\mathrm{PAD}^{(21)}$.

Durante as quatro semanas, as participantes receberam orientação sobre a execução adequada de cada exercício. Em seguida, completaram duas sessões de familiarização com duas séries de 12 a 15 repetições submáximas nos exercícios leg press $45^{\circ}(\cong 40 \mathrm{~kg})$, supino $(\cong 10 \mathrm{~kg}) \mathrm{e}$ rosca bíceps $(\cong 6 \mathrm{~kg})$. Depois, os testes de $1 \mathrm{RM}$ foram realizados em quatro dias separados, com 10 minutos entre os exercícios, pelo mesmo avaliador, segundo descrições recomendadas ${ }^{(22)}$.

OTF consistiu em 13 exercícios alternados por segmento superior e inferior, na seguinte ordem: leg press $45^{\circ}$, supino reto com barra, extensão de joelhos, remada baixa, extensão do cotovelo no cabo, flexão dos joelhos, rosca bíceps em pé, flexão plantar na barra guiada, cadeira adutora, cadeira abdutora e duas séries de 20 a 30 repetições de exercícios abdominais. Ele apresentou duração de 13 meses, com uma frequência semanal de duas vezes (segunda e quinta-feira), realizando três séries de 8 a 12 repetições máximas (812RM) em cada exercício, com duração de cada repetição de 3 a 4 segundos. As intensidades de treinamento eram readequadas a cada sessão, a fim de garantir a zona de RM.

Coletaram-se as amostras sanguíneas $(3 \mathrm{~mL})$ da veia antecubital em tubos a vácuo para centrifugação a 2.500 rpm, a $4^{\circ} \mathrm{C}$, durante 20 minutos; em seguida, separou-se o soro em alíquotas de $500 \mu \mathrm{L}$, armazenando-o em freezer a $-80^{\circ} \mathrm{C}$ até o momento das análises. O método ELISA determinou a dosagem de resistina, de acordo com as especificações do Kit de Alta Sensibilidade RayBio ${ }^{\circledR}$ (RayBiotech, Inc, Norcross, GA, USA). Para garantir a precisão dos resultados, todas as dosagens foram determinadas em duplicata e apresentadas em $\mathrm{pg} / \mathrm{mL}$.

No processamento estatístico, todos os dados foram expressos em média \pm desvio padrão (DP). Inicialmente, realizou-se os testes de Shapiro Wilk (normalidade) e Levene, seguidos de ANOVA (com medidas repetidas), para as comparações entre os momentos Pré, 6 meses e 13 meses. Para avaliar as possíveis correlações entre resistina $\times$ pressão arterial, resistina $\times$ força muscular e força $\times$ pressão arterial, utilizou-se o teste de correlação de Pearson, considerando-se a seguinte classificação: nula $=0,0$; fraca $=0,01$ a 0,3 ; regular $=0,31$ a 0,6 ; forte $=0,61$ a 0,9 ; muito forte $=0,91$ a 0,99 ; e plena $=1,0^{(23)}$. Em todos os cálculos, foi fixado um nível crítico de $5 \%(\mathrm{p}<0,05)$.

As participantes assinaram o Termo de Consentimento Livre e Esclarecido, conduzindo-se o estudo de acordo com a Resolução 196/96 do Conselho Nacional de Saúde (CNS), recebendo aprovação do Comitê de Ética em Pesquisa com Seres Humanos do Centro Universitário Central Paulista UNICEP (Protocolo 042/2008 do CEP/UNICEP).

\section{RESULTADOS}

Após 13 meses do programa de $\mathrm{TF}$, não foram observadas alterações significativas na massa corporal $(67,5 \mathrm{~kg} \pm 10,8$ versus $66,5 \mathrm{~kg} \pm 10,9)$ nem no índice de massa corporal $\left(28,0 \mathrm{~kg} / \mathrm{m}^{2} \pm 4,9\right.$ versus $27,5 \mathrm{~kg} / \mathrm{m}^{2} \pm$ $4,5)$. No entanto, houve redução significativa da PAS aos 6 $(-3,6 \mathrm{mmHg})$ e $13(-4,8 \mathrm{mmHg})$ meses, se comparados ao 
momento Pré (Figura 1). Não houve diferença significativa nos valores de PAD, PAM e PP nos momentos avaliados (Figura 1).

Houve redução significativa na concentração sorológica de resistina em todos os momentos (Pré $\times 6$ meses; Pré $\times$ 13 meses; 6 meses $\times 13$ meses) analisados (Tabela I). A redução foi de $46 \%$ após 13 meses de treinamento.

Observou-se aumento significativo da força muscular no leg press $45^{\circ}$, supino e flexão de cotovelo (Tabela II) em todos os momentos (Pré $\times 6$ meses; Pré $\times 13$ meses; 6 meses $\times 13$ meses). $\mathrm{O}$ aumento foi de $98,5 \%$ no leg press $45^{\circ} ; 29,6 \%$ no supino e $26,5 \%$ na rosca bíceps após os 13 meses.

A Tabela III demonstra os coeficientes de correlação entre as variáveis resistina, PAS e força muscular. Observase correlação positiva muito forte entre resistina $\times$ PAS; e negativa muito forte para resistina $\times$ força muscular e força muscular $\times$ PAS.

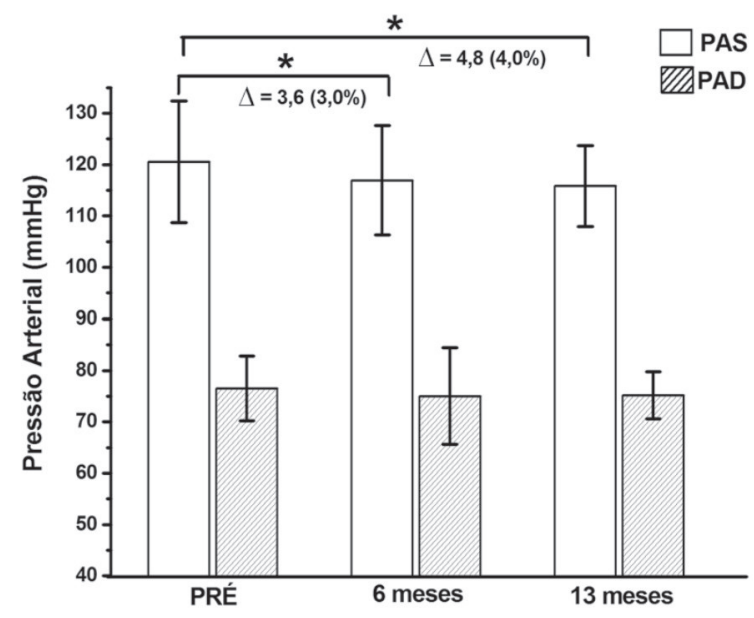

Figura 1 - Valores de pressão arterial (média \pm DP, n=23) nos momentos Pré, 6 meses e 13 meses do programa de treinamento de força. Pré: momento pré-treinamento; PAS: pressão arterial sistólica; PAD: pressão arterial diastólica. $\Delta$ : valor absoluto de redução comparado ao momento Pré. * $\mathrm{p}<0,05$ comparado ao momento Pré.

Tabela I - Concentração plasmática de resistina nos momentos Pré, 6 meses e 13 meses do programa de treinamento de força.

\begin{tabular}{cccc}
\hline & Pré & 6 meses & 13 meses \\
\hline Resistina $(\mathrm{pg} / \mathrm{ml})$ & $30272,49 \pm 8100,15$ & $20114,52 \pm 4416,50^{*}$ & $16350,62 \pm 2404,67^{*} \#$ \\
\hline
\end{tabular}

Os dados são apresentados como média \pm DP $(n=23)$. Pré: avaliação antes do início do programa de treinamento de força; * $\mathrm{p}<0,01$ comparado ao momento Pré. \# p<0,01 comparado ao momento 6 meses. Teste ANOVA (medidas repetidas).

Tabela II - Força máxima nos momentos Pré, 6 meses e 13 meses do programa de treinamento de força.

\begin{tabular}{lccc}
\hline Exercícios & Pré & 6 meses & 13 meses \\
\hline Leg press $45^{\circ}(\mathrm{kg})$ & $172,35 \pm 27,35$ & $226,96 \pm 31,36^{*}$ & $348,64 \pm 40,82^{*} \#$ \\
Supino $(\mathrm{kg})$ & $31,95 \pm 4,18$ & $38,73 \pm 6,03^{*}$ & $41,80 \pm 5,61^{*} \#$ \\
Flexão do cotovelo $(\mathrm{kg})$ & $21,00 \pm 2,43$ & $23,31 \pm 2,67^{*}$ & $26,56 \pm 2,91^{*} \#$ \\
\hline
\end{tabular}

Os dados são apresentados como média \pm DP ( $n=23)$. Pré: avaliação antes do início do programa de treinamento de força; * $\mathrm{p}<0,01$ comparado ao momento Pré. \# p<0,01 comparado ao momento 6 meses. Teste ANOVA (medidas repetidas). 
Tabela III - Coeficiente de correlação entre as variáveis fisiológicas de repouso.

\begin{tabular}{lccc}
\hline Correlações & r & Classificação* & Valor de p \\
\hline Resistina $\times$ Pressão Arterial Sistólica & 0,99 & Muito forte & 0,02 \\
Resistina $\times$ Força Muscular & $-0,98$ & Muito forte & 0,01 \\
Força Muscular $\times$ Pressão Arterial Sistólica & $-0,97$ & Muito forte & 0,01 \\
\hline
\end{tabular}

r: coeficiente de correlação de Pearson. *Classificação do coeficiente de correlação segundo Albuquerque et al. (2010).

\section{DISCUSSÃO}

O objetivo do presente estudo foi verificar os efeitos crônicos (13 meses) do TF sobre as variáveis hemodinâmicas e a concentração sorológica da resistina em mulheres na pós-menopausa. A hipótese foi parcialmente confirmada, sendo observada uma redução clinicamente significativa nos valores da PAS e nos níveis sorológicos da resistina, porém, nenhuma alteração nos valores da PAD, PAM e PP após 13 meses de TF. Adicionalmente, houve forte correlação entre PAS $\times$ resistina $\times$ força muscular. Como esperado, o programa de TF aumentou a força muscular dos grupos musculares avaliados.

Com relação ao controle da pressão arterial, o American College of Sports Medicine ${ }^{(24)}$ e o American Heart Association ${ }^{(25)}$ recomendam o TF como fator de prevenção e/ ou terapia não medicamentosa às doenças cardiovasculares para mulheres e idosos. No presente estudo, houve redução de 4,8 mmHg nos valores da PAS e manutenção da PAD (4\% e $2 \%$, respectivamente) de repouso após 13 meses de TF. Esses resultados corroboram com os achados de uma meta-análise ${ }^{(26)}$, que encontrou redução de $2 \%$ e $4 \%$ nas PAS e PAD, respectivamente. Em outra meta-análise, de nove estudos controlados e aleatórios, observou-se redução de 3,2 e 3,5 mmHg na PAS e PAD, respectivamente ${ }^{(27)}$. Mesmo sendo pequena a redução da PAS observada nos estudos citados, ela é clinicamente importante e fortemente relacionada à diminuição de $5 \%$ a $9 \%$ na morbidade cardíaca, $8 \%$ a $14 \%$ na ocorrência de acidente vascular encefálico e $4 \%$ na mortalidade por todas as causas ${ }^{(28)}$.

Dentre os mecanismos fisiológicos envolvidos no controle da pressão arterial, pode-se destacar o número e o calibre de pequenas artérias e arteríolas com um papel fundamental no controle da resistência periférica total e $\mathrm{PAM}^{(21)}$. Na prática clínica, a pressão arterial é definida com os valores da PAS e PAD. No entanto, uma análise mais detalhada da curva da pressão arterial deve considerar a somatória do componente médio - a PAM - e o componente pulsátil - a PP. A PAM é a pressão para a distribuição adequada do fluxo e oxigênio para os tecidos e órgãos, sendo dependente, principalmente, do débito cardíaco e da resistência vascular periférica. A PP é caracterizada pelo papel das grandes artérias de minimizar a pulsatilidade e depende da ejeção ventricular, da rigidez arterial e do tempo de reflexão da onda ${ }^{(29)}$. A PAM e a PP estão associadas ao controle adequado da pressão arterial e a fatores independentes de risco cardiovascular, especialmente para a mortalidade cardíaca em mulheres entre 50 e 60 anos de idade ${ }^{(21)}$. Assim, fica evidente a importância clínica do atual estudo, que, além da redução da PAS, promoveu a manutenção da PAM e PP durante 13 meses do programa de TF.

Evidências indicam que o envelhecimento é acompanhado pelo aumento nos níveis séricos de diferentes mediadores inflamatórios, como a proteína $\mathrm{C}$ reativa, fator de necrose tumoral-alfa, interleucina-6, interleucina1beta e resistina, os quais são associados à fisiopatologia da hipertensão arterial sistêmica ${ }^{(30-32)}$. A resistina é um marcador de estado inflamatório e tem sido relacionada ao desenvolvimento de doenças cardiovasculares ${ }^{(5,6,7)}$. Ademais, há uma correlação negativa entre a atividade física e os níveis de mediadores inflamatórios circulantes ${ }^{(33)}$.

Identificou-se uma associação entre os níveis de resistina, a inflamação senil e as disfunções vasculares com o aumento da incidência de hipertensão em mulheres acima de 55 anos na pós-menopausa ${ }^{(5)}$. No presente estudo, observou-se uma redução de $46 \%$ na concentração sorológica de resistina e uma forte correlação entre PAS e resistina após 13 meses de TF. Portanto, o TF pode contribuir com esse grupo de mulheres na pós-menopausa para a redução do quadro inflamatório senil e do risco cardiovascular.

Os dados de uma investigação( ${ }^{(34)}$ confirmam os resultados do presente estudo em relação ao aumento de força muscular. Foi demonstrado aumento na resistência e velocidade da marcha, na capacidade para subir escadas e equilíbrio dinâmico, com consequente prevenção de quedas $^{(28)}$. Também se observou redução dos níveis de resistina sérica após 16 semanas de TF em mulheres na pós-menopausa ${ }^{(10)}$. Um dos aspectos a serem destacados no presente estudo é que a redução da resistina sérica, bem como o aumento da força muscular, ocorreu após 6 e 13 meses de treinamento.

A forte relação inversa entre a PAS e a força muscular foi outro resultado interessante. Estudos prévios com resultados similares foram realizados em mulheres 
sedentárias de meia idade ${ }^{(14,15)}$. Além disso, tem sido demonstrada maior incidência de hipertensão arterial em homens com baixa força muscular ${ }^{(35)}$, e de mortalidade em homens hipertensos de meia idade e idosos com baixa força muscular ${ }^{(36)}$. Uma possível relação benéfica entre o aumento da força muscular e a redução da pressão arterial pode ser explicada pela diminuição da complacência arterial periférica, a qual ocorre durante exercícios que necessitam de força muscular. Essa alteração na complacência é um efeito protetor de longo prazo, proporcionado por mudanças no conteúdo do músculo liso das paredes arteriais e nas propriedades de colágeno e elastina, que, em parte, reduzem a pressão arterial em repouso ${ }^{(37)}$.

Os resultados do presente estudo devem ser interpretados com cautela, visto que existem nele algumas possíveis limitações metodológicas. Primeiro, o número relativamente pequeno de voluntárias dificulta a generalização e aplicação dos resultados para a população em geral. Segundo, a ausência do grupo controle, que não permite comparações efetivas dos resultados obtidos de um grupo que não realizou o treinamento. Entretanto, tem sido especulado que um grupo controle não é sempre necessário, especialmente considerando que, nessa faixa etária, a saúde pode ser comprometida em virtude do sedentarismo $^{(10-12,25,33)}$. Além disso, os resultados de um grupo controle não apresentam efeito positivo sobre a força muscular e os fatores de risco cardiovascular ${ }^{(38-40)}$.

\section{CONCLUSÃO}

Em conclusão, o TF durante 13 meses promoveu aumento da força muscular, redução de resistina e da pressão arterial sistólica das pacientes investigadas. Essas adaptações são fundamentais para a redução do risco de desenvolvimento de doenças e eventos cardiovasculares em mulheres pós-menopáusicas, ressaltando a importância clínica do TF nesta população.

\section{REFERÊNCIAS}

1. Martins CB, Primolan LV. A importância para educação na evolução da mulher no mercado de trabalho globalizado [acesso em 2011 Ago 4]. Disponível em: $\mathrm{http} / /$ :www.uninove.br.

2. Rosano GMC, Vitale C, Fini M. Cardiovascular aspects of menopausal hormone replacement therapy. Climacteric. 2009;12(Suppl 1):41-6.

3. Tremollieres F, Ribot C. Bone mineral density and prediction of non-osteoporotic disease. Maturitas. 2010;65(4):348-51.
4. Coylewright M, Reckelhoff JF, Ouyang P. Menopause and hypertension: an age-old debate. Hypertension. 2008;51(4):952-9.

5. Zhang L, Curhan GC, Forman JP. Plasma Resistin Levels Associate RisK For Hypertension among Nondiabetic Women. J Am Soc Nephrol. 2010;21(7):1185-91.

6. Lee SE, Kim HS. Human resistin in cardiovascular disease. J Smooth Muscle Res. 2012;48(1):27-35.

7. Ding Q, White SP, Ling C, Zhou W. Resistin and cardiovascular disease. Trends Cardiovasc Med. 2011;21(1):20-7.

8. Shiguemoto GE, Botero JP, Prestes J, Ázar LMB, Marin $\mathrm{CT}$, Monaretti FH, et al. De bem com a vida: programa de intervenção preventiva e promoção de saúde e qualidade de vida em mulheres pós-menopáusicas por meio de treinamento de força periodizado. ABMES Cad. 2008;19(1):11-39.

9. Leite RD, Prestes J, Pereira GB, Shiguemoto GE, Perez SE. Menopause: highlighting the effects of resistance training. Int J Sports Med. 2010;31(11):761-7.

10. Prestes J, Shiguemoto GE, Botero JP, Frollini A, Dias R, Leite $\mathrm{R}$, et al. Effects of resistance training on resistin, leptin, cytokines and muscle force in elderly postmenopausal women. J Sports Sci. 2009;27(14):160715.

11. Frontera WR, Meredith CN, O'Reilly KP, Knuttgen HG, Evans WJ. Strength conditioning in older men: skeletal muscle hypertrophy and improved function. J Appl Physiol. 1988;64(3):1038-44.

12. Braith RW, Stewart KJ. Resistance exercise training: its role in the prevention of cardiovascular disease. Circulation. 2006;113(22):2642-50.

13. Tibana RA, Teixeira TG, De Farias DL, Silva AO, Madrid B, Vieira A, et al. Relação da circunferência do pescoço com a força muscular relativa e os fatores de risco cardiovascular em mulheres sedentárias. Einstein (São Paulo). 2012;10(3):329-34.

14. Tibana RA, Balsamo S, Prestes J. Associação entre força muscular relativa e pressão arterial de repouso em mulheres sedentárias. Rev Bras Cardiol. 2011;24(3):163-8.

15. Tibana RA, César D, Tajra V, Vieira A, Franz CB, Pereira GB, Prestes J. Avaliação da pressão arterial em mulheres sedentárias e sua relação com a força muscular. Rev Bras Promoç Saúde. 2012; 25(3):33743. 
16. Tibana RA, Tajra V, César D, De Farias DL, Teixeira TG, Prestes J. Comparação da força muscular entre mulheres brasileiras com e sem síndrome metabólica. ConScientiae Saúde. 2011;10(4);708-14.

17. Farias DL, Tibana RA, Teixeira TG, Vieira DCL, Tajra V, Nascimento DC, et al. Idosas com síndrome metabólica apresentam maior risco cardiovascular e menor força muscular relativa. Einstein (São Paulo), 2013. No prelo.

18. Newman AB, Kupelian V, Visser M, Simonsick EM, Goodpaster BH, Kritchevsky SB. Strength, but not muscle mass, is associated with mortality in the health, aging and body composition study cohort. J Gerontol A Biol Sci Med Sci. 2006;61(1):72-7.

19. Stewart LK, Flynn MG, Campbell WW, Craig BA, Robinson JP, Timmerman KL, et al. The influence of exercise training on inflammatory cytokines and C-reactive protein. Med Sci Sports Exerc. 2007;39(10):1714-9.

20. Sociedade Brasileira de Cardiologia-SBC, Sociedade Brasileira de Hipertensão-SBH, Sociedade Brasileira de Nefrologia-SBN. V Diretrizes Brasileiras de Hipertensão Arterial. Arq Bras Cardiol. 2007;89(3):e24-79.

21. Yoshitomi Y, Nagakura C, Miyauchi A. Significance of mean blood pressure for blood pressure control. Int Heart J. 2005;46(4):691-9.

22. Brown LE, Weir JP. Procedures recommendation I: accurate assessment of muscular strength and power. J Exerc Physiol. 2001;4(3):1-21.

23. Albuquerque PL, Quirino MAB, Santos HH, Alves SB. Interferência da prática de atividade física habitual na postura de jovens. Rev Ter Man. 2010;8(37):198-203.

24. Pescatello LS, Franklin BA, Fagard R, Farquhar WB, Kelley GA, Ray CA. American College of Sports Medicine position stand: exercise and hypertension. Med Sci Sports Exerc. 2004;36(3):533-553.

25. Pollock ML, Franklin BA, Balady GJ, Chaitman BL, Fleg JL, Fletcher B, et al. AHA Science Advisory. Resistance exercise in individuals with and without cardiovascular disease: benefits, rationale, safety, and prescription. An advisory from the Committee on Exercise, Rehabilitation, and Prevention, Council on Clinical Cardiology, American Heart Association; Position paper endorsed by the American College of Sports Medicine. Circulation. 2000;101(7):828-33.

26. Kelley GA, Kelley KS. Progressive resistance exercise and resting blood pressure - a meta-analysis of randomized controlled trials. Hypertension. 2000;35(3):838-43.

27. Cornelissen VA, Fagard RH. Effect of resistance training on resting blood pressure: a meta-analysis of randomized controlled trials. Hypertension. 2005;23(2):251-9.

28. Williams MA, Haskell WL, Ades PA, Amsterdam EA, Bittner V, Franklin BA, et al. Resistance exercise in individuals with and without cardiovascular disease. Circulation. 2007;116(5):572-84.

29. Bos WJW, Verrij E, Vincent HH, Westerhof BE, Parati G, van Montfrans GA. How to assess mean blood pressure properly at the brachial artery level. J Hypertension. 2007;25(4):751-5.

30. Giunta S. Is inflammaging an auto[innate]immunity subclinical syndrome? Immun Ageing. 2006;12(3):1-2.

31. Berggren JR, Hulver MW, Houmard JA. Fat as endocrine organ: influence of exercise. J Appl Physiol. 2005;99(2):757-764.

32. Silva AO, Tibana RA, Karnikowski MGO, Funghetto SS, Prestes J. Inflammatory status in older women with and without metabolic syndrome: is there a correlation with risk factors? Clin Interv Aging. 2013;8:361-7

33. Nicklas BJ, Brinkley TJ. Exercise Training as a Treatment for Chronic Inflammation in the Elderly. Exerc Sport Sci Rev. 2009;37(4):165-170.

34. Fiatarone MS, Rhonda O, Jacqui R. Efficacy of progressive resistance training on balance performance in older adults: a systematic review of randomized controlled trials. Sports Med. 2008;38(4):317-43.

35. Maslow AL, Sui X, Colabianchi N, Hussey J, Blair SN. Muscular strength and incident hypertension in normotensive and prehypertensive men. Med Sci Sports Exerc. 2010;42(2):288-95.

36. Artero EG, Lee DC, Ruiz JR, Sui X, Ortega FB, Church $\mathrm{TS}$, et al. A prospective study of muscular strength and all-cause mortality in men with hypertension. J Am Coll Cardiol. 2011;57(18):1831-1837.

37. Bertovic DA, Waddell TK, Gatzka CD, Cameron JD, Dart AM, Kingwell BA. Muscular strength training is associated with low arterial compliance and high pulse pressure. Hypertension. 1999;33(6):1385-91.

38. Kadoglou NP, Perrea D, Illiadis F, Angelopoulo N, Liapis C, Alevizos M. Exercise Reduces Resistin and Inflammatory Cytokines in Patientes with Type 2 Diabetes. Diabetes Care. 2007:30(3):719-21. 
39. Wood RH, Reyes R, Welsch MA. Concurrent Cardiovascular and resistance training in healthy older adults. Med Sci Sports Exerc. 2001;33(10):1751-8.

40. Roelants M, Delecluse C, Verschueren SM. Wholebody-vibration training increases knee-extension strength and speed of movement in older women. J Am Geriatr Soc. 2004;52(6):901-8.
Endereço para correspondência:

Gilberto Eiji Shiguemoto

Universidade Federal de São Carlos - UFSCar

Laboratório de Fisiologia do Exercício

Rodovia Washington Luis, Km 235

CEP: 13.565-905 - São Carlos - SP

E-mail: gileiji@terra.com.br 